\title{
The Dynamics of Google within the Frame of a Large Technical System - An LTS analysis of Google
}

\author{
Julian Schäfer 1
}

\begin{abstract}
The Large Technical System approach was introduced by the influential historian of technology, Thomas P. Hughes, in the 1970's and is one of the most prominent theoretical frameworks within the Science and Technology Studies. However, it has found little attention in relation to the digital realm. This research applies the LTS framework onto the US-American company Google and seeks to bring a conceptual understanding to the company's exponential growth. Thus, it describes the emergence and evolution of Google as a complex system - an alignment of components of technical and non-technical nature - and assigns patterns and concepts to its development. This research provides an answer to how Google not only gained a system structure but also reached the notion of momentum. Yet, suggesting a social constructivist path, this paper secludes by elucidating the influencing power of the LTS's user - an important factor which was widely disregarded in the initial works of Hughes.
\end{abstract}

Keywords: Large Technical System, Google, System Builders, Momentum, Transfer

\section{The Dynamics of Google within the Frame of a Large Technical System}

Thomas Edison invented the light bulb, founded the Edison General Electric Company and brought light to American cities and villages (Hughes, 1993). Henry Ford launched the Quadricycle, established the Ford Motor Company, and brought the car to American streets (Hughes, 2000). Larry Page and Sergey Brin brought to life a search algorithm, started Google Inc. and changed information gathering on a global scale. (Vise \& Malseed, 2006). In modern society, only a few companies had and have a ubiquitous influence on people's life, culture and politics. In most cases, they stared out with small, unique ideas and, within a couple of years, developed into systems and networks of great structural complexity. (Joerges, 1996)

Google, even more than General Electric and Ford, is one of the leading examples of companies which rose from a simple idea into a multi-billion-dollar business-network. It took Edison more than half a century to electrify American homes (Hughes, 1993). In only a fragment thereof - 9 years - Google reached 70 percent of all internet users, billions of people across the world. (Auletta, 2010). Although this comparison lacks considering the difference between the physical and the digital realm, it encapsulates the enormous scale of growth. With a fourth term revenue of $\$ 39,2$ billion Dollar in 2018 (De Vynck, 2019) it is almost unimaginable that the history of Google started in a garage only two decades ago. But how did the company arrive at such a point of complexity, no longer existing as a pure search engine, but as a company that has branches in sectors ranging from biology to robotics? (Schulz, 2017)

Being such a young company, not much academic research has been done on Google's emergence and growth. By approaching its business model (Schmidt, 2014; Holden, 2008; Lowe, 2009) or the structure of algorithm (Baskin, Brashars \& Long 2007; Burns \& Sauers, 2014; Guermeur \& Unruh, 2010) most publications deal with narrow aspects of Google's characteristics. The history of Google and especially

1 Julian Schäfer received a bachelor degree in Arts and Culture at Maastricht University in 2019. At the moment he takes a Master in Governance of Technology and Innovation at RWTH Aachen University. Contact: julianjschaefer@web.de lee 
its formation is only described in the books by Vise \& Malseed (2006) and Auletta (2010), both taking a narrative approach - constructing a chronological sequence of events and weaving them in a congruent manner.

Yet, it is not the only way to describe the formation of a company. By approaching its development from a larger system perspective, historians and predominantly scholars of Science and Technology Studies (STS), aim at finding identifiable and recurring patterns and concepts of evolution. In doing so, they construct a narrative too but base it on a conceptual framework. Arguably, one of the most prominent and recent attempts within STS is the Large Technical System (LTS) approach. The general framework of an LTS was introduced by the American historian of technology Thomas P. Hughes (1993) in the book "Networks of Power" in which he analyzed the emergence of electricity systems from 1880 to 1930 on a regional and national level. The approach found large appreciation and, in addition to the named examples of General Electric and Ford, was applied to a variety of other fields such as railway networks (Kaijser 2005) or water transport systems (Odhnoff, \& Svedin, 1998). What makes this approach special is the description of the successful alignment of technical and non-technical elements into a sociotechnical whole. This implies, physical, technical artifacts such as inventions and machinery, but also human components such as inventors or managers who guide the development of a company fall under the same category. Additionally, Hughes assigns "loosely defined" patterns and concepts onto the development processes which can be identified and analyzed. In doing so, Hughes illuminates how companies gain a system structure and a structuring capacity onto their larger environment (Bijker, Hughes, Pinch, 2012).

It is quite evident that the current state of Google does possess a system structure and structuring capacity as described by Hughes. In 2015, Google was divided into the company's traditional and new branches. "Google search"," YouTube", "Google Maps", inter alia, are now separated from new projects such as "Google $X^{\prime \prime}$ and "Google Ventures" which conduct research in the field of autonomous cars and microbiology. All companies are united under the umbrella company called "Alphabet" (Schulz, 2017). No other company seems to have such an omnipresent role in everyday life. Google is no longer the simple search engine it was originally intended to be. It has become part of everyday life, culture and language. "The internet made information available. Google made information accessible" (Schulz, 2017 p.35). In this context, it is of greater interest to take a step back and investigate how such a structure of power came into existence and what it may imply.

This study entails applying an LTS approach to Google. The research investigates if phases and concepts as described by Hughes can be identified in Google's history. I am interested in finding out to what extent the theoretical concept of Large Technical Systems (LTS) can provide fruitful insights into the complexity and success of this internet platform? In doing so, I will analyze if the company became part of what Hughes calls a "deep structure", i.e. a structure that has surpassed key drivers of social change such as politics and geography. No previous LTS research considered an internet platform as the object of study. The majority of previous LTS studies conducted research with individual companies in individual countries. (Kaijser \& Vleuten, 2006) I do not intend to cover the entire history of Google. For the analysis, I will spotlight the company's early period (1997 - 2005), with special attention to the year 2002. In this year, Google first became financially profitable due to the introduction of personalized advertising. I will furthermore focus on the major events in the company's history and restrict myself to the general LTS definition by Hughes. The information about Google's development are predominantly taken from the books "The Google story" (Vise \& Malseed, 2006); "Googled: The end of the world as we know it" (Auletta, 2010), "Was Google wirklich will. Wie der einflussreichste Konzern der Welt unsere Zukunft verändert" 
(Schulz, 2017); "Google: How google works" (Schmidt, 2014) as well as a variety of newspaper articles. Combined, these sources provided me comprehensive insights into its chronological emergence. Further definitions of subsequent LTS research that focus on narrow aspects of the system will only be regarded to a limited extent. In the first step of my research, I will elucidate the Large Technical System approach and apply it to the corporate history of Google. Furthermore, I will discuss the framework under consideration of a previously widely disregarded actor, namely the user. Overall, this research conceptualizes the development of Google for the first time within the realm of STS. In doing so, I intend to pave the way for further discussion and research in the field of LTS studies in relation to modern internet companies.

\section{Large Technical Systems - A Versatile Interpretable Approach}

Large Technical Systems are complex and so is their definition. It is immensely difficult to use a generally accepted definition for LTS research. Unlike, for instance, the social construction of technology (SCOT) by Pinch and Bijker, the LTS approach does not supply a sharply defined methodology (Bijker, Hughes, Pinch, 2012). Rather, it should be interpreted as an open research approach with different concepts and research strategies (Vleuten \& Kaijser 2006). Researchers applying the Large Technical System approach use definitions inspired by the work of Hughes but substitute them to fit their specific needs and purposes. Therefore, even the meaning of the basic terms "large", "technical" and "system" can vary strongly depending on their discipline. One attempt is to define a Large Technical System by its function suggesting to "determine the relative quantity (complexity, speed, rate of growth, etc.) of activities materialized in such systems and the quantity of other social processes necessitated in order to function" (Mayntz \& Hughes, 2005 p.24).

Other definitions depend on the relative largeness of the system per se. Particularly, Bernward Joerges defines Large Technical systems as:

[...] complex and heterogeneous systems of physical structures and complex machineries which (1) are materially integrated, or "coupled" over large spans of time, quite irrespective of their particular cultural, political, economic and corporate makeup, and (2) support or sustain the function of very large numbers of other technical systems, whose organizations they thereby link. (Mayntz \& Hughes, 2005 p.24)

Although such definitions contribute to a basic understanding of the subject, they are of little use for an extensive discussion of LTSs in relation to Google. To simply apply these definitions to internet service provider would undercut its broader dynamics, such as the implications for society at large. Additionally, the realm of my case study would turn out to be problematic to apply in this context since Google's evolution takes place in the digital and physical sphere. Such factors were previously disregarded. Unfortunately, Hughes, who died during the during the rise of the most popular internet services in 2005 (Ryan, 2013), did not explicitly apply his LTS approach on digital service providers. Yet, his methodological angle is most applicable for my approach. Instead of providing a sharp, five-line working definition he analyzed the dynamics from a descriptive stance and organized the system in different concepts. In the following, I will illustrate Hughes' basic concepts of LTSs. Simultaneously, I will here relate to the development of Google and point out corresponding patterns. 


\section{Large Technical System}

\subsection{Structure}

Before diving into the analysis of Google, I need to evaluate Hughes' fundamental framework of LTSs in more depth. The fundamental structure of an LTS consists of a network of interrelated components - the system. The system is of national, but mostly global scale (Gökalp, 1992). Noticeably, components have different functions and can be of very different nature (Bijker, Hughes, Pinch, 2012). Thus, Hughes distinguished between physical artifacts - material technology components - such as machines and production devices, organizational structures which control and govern the system, as well as institutional elements which provide a regulatory framework, the system's cultural values and professional know-how. Together the components build up a material and immaterial as well as human entity with closely interrelated parts and processes. Hughes stresses that a clear classification and restriction of components must be provided. If one component is investigated in isolation, a system structure with further components and organizational arrangements might appear again. In other words, components can consist of individual sub-systems and are categorized in accordance with the system. All components are united by a common objective - the system goal. They all contribute to a larger outcome or product.

At this point, scholars of STS might point out similarities to the "Actor-Network" approach by Michel Callon and Bruno Latour in which components - or according to Callon and Latour, "actors" - can be of technical and physical nature, too (Muniesa, 2015). To a certain extent, this is correct, but while the "Actor-Network" approach treats actors of the network with relative equality, the LTS approach argues for a guiding force which steers the LTS. According to Hughes, such forces are executed by managers, inventors, engineers called system builders (SB). The SB aligns the technical and non-technical components into a socio-technical whole. In other words, the SB oversees the hardware invention and development as well as the organizational structure of the Large Technical System and enforces centralization within. The character of the SB varies in accordance to the systems development. Hence, Hughes distinguishes between the inventor-entrepreneur, the manager-entrepreneur and the financeentrepreneur. Even though the System Builder seems to be a dominant figure, the system and the SB are strongly dependent on each other. While the SB is reliant on the system's function and the system is reliant on the SB's strategy. In my later analysis, I will look as the concept of the system builder more closely.

Taking a step back, the Large Technical System is placed in an environment - a physical but also non-physical setting such as the market - which cannot be controlled by the system builder. However, it is the goal of the system and its builder to gain control over some of the environmental factors by incorporating them into the system. For instance, this can be achieved through the domination of the market. By means of control, factors of uncertainty such as a once free market are eradicated. The LTS becomes more calculable and more influential in this environment. Later Hughes describes this phenomenon as the momentum, a concept which will be relevant in the last part of the paper.

\subsection{Emergence and Development}

Having explained the foundation of the system, I can turn to the LTS's emergence and development. Hughes suggests that the development of a large technical system is neither linear nor stable at any time (Bijker, Hughes, Pinch, 2012). It undergoes a constant process of change. New Large Technical Systems can supersede others or merge. They can spread from national to international territorial or change

\footnotetext{
Marble

4 Research

Papers
} 
radically in competition with others. However, changes of an LTS can be framed into four major phases: invention phases, development phase, innovation phase and competition and consultation.

While going thought these phases, the system's number of interrelated components increases, and consequently, does its complexity. The expansion, however, comes with some cost and hence sooner or later the LTS will inevitably encounter problems which hinder its further growth. Hughes calls these problems reverse salients and they are characterized by underdeveloped components, organizational anomalies or general uneven development. To illustrate, a technical system's growth might be impeded by environmental factors, restricting policies or by a system's component becoming overwhelmed by its current task. It is the duty of the system builder to identify reverse salients, characterize them as critical problems and enact countermeasures. Put simply, the system builder is the "problem-solving manager" who steers the direction of an LTS. Like the system's components, the reverse salient can be of technical and non-technical character. Therefore, the system builder often engages in transdisciplinary problemsolving.

\subsection{Invention Phase}

A new Large Technical System originates in the invention phase. According to Hughes, all sociotechnical systems start out with and are structured around a technical core, the so called radical new invention. (Bijker, Hughes, Pinch, 2012) The radical new invention is created by the inventor entrepreneur. Hughes describes radical new inventions as technical solutions to a problem which however cannot be integrated into an existing Large Technical System. To illustrate, Thomas Edison invented the light bulb, one of the most revolutionary technical artifacts of the 19th century. But, since no electricity network existed at the time, there was no possibility for other LTS - mostly production companies - to integrate the light bulb as component into their systems. The idea was rejected. (Hughes, 1993). Hughes suggests that radical new inventions can either occur within or outside of an LTS. Both options are characterized by two different types of inventors. The independent inventor entrepreneur creates from an external position, outside of the LTS. He has complete freedom from any organizational constrains. On the one hand, he does not rely on contributing to an LTS due to solving its reverse salient, on the other hand, he lacks important resources for the invention process. The professional inventor entrepreneur, on the contrary, works for a Large Technical System. He has access to a variety of resources, however, lacks creative freedom because the creation of new inventions, which are incompatible with the existing LTS structure, are not favored by the system. In effect, radical new inventions do not occur on a regular basis. They are extremely rare because of their demand for innovative freedom and resources. (Bijker, Hughes, Pinch, 2012)

For the analysis of the innovation phase of Google, the environment in which the company took shape needs to be considered. Unlike the Ford Motor Company or General Electrics, Google is not a producer of physical goods in a physical environment, but a service provider in a digital realm. For this reason, my LTS analysis has conceptualized the internet per se as environment. In a different analytical frame, the internet as such can be considered a Large Technical System itself, yet since it provides the stage for Google's and other relevant companies' rises, I regard this reduction as justified.

In the early 90's, the internet differed heavily from how we know it today. People were just beginning to write e-mails or create websites. (Vise \& Malseed, 2006). Bit by bit actors of every kind became aware that almost silently a new marketplace was on the rise: The World Wide Web. Yet, despite all its new advantages, one fundamental problem soon emerged. Although it suddenly became possible to 
create information and share with everyone in the world, the more the internet was used, the more difficult it became to find what one was looking for. Efficient web-searching was not born yet.

Google's innovation phase started due to the two system builders named Larry Page and Sergey Brin. Page and Brin were both PhD students at Stanford University and had a common interest in the emerging fields of data science and data mining. They were interested in improving internet searching. Web-searchprograms at the time were almost useless. With the exponential growth of the web, search engines as for example offered by Netscape, which was the most popular web browser of the early World Wide Web, crawled every website word by word and hence needed a huge amount of time to digest and publish results. Inktomi, another example for former popular search engines, could not even find itself if its name was typed into the search-bar. Most search engines came up with thousands of results, completely unsorted and mostly without anticipated results. Even Yahoo, one of the first internet platforms which provided a search function, went so far to employ human editors to improve their search.

The solution which would bring order to chaos was proposed by Page and Brin in the form of a radical new invention called "Page Rank". "Page Pank" was an algorithm which not only came up with the correct search results but also seemed to sort them according to their relevance (Wiesend, 2015). But, how did it work? Until today, the precise function of Google's backbone remains secret. However, to understand Google's radical new invention in its most essential one must understand a different search engine - arguably Google's inspiration -, namely Altavista. Instead of crawling through the entire web, searching for buzz words, Altavista used links - highlighted words in a text document which led the reader directly to a web-page - to generate search results. The procedure allowed the search engine to be incredibly fast, especially in comparison with its competitors. However, also Altavista shared the problem of not sorting the results in a useful way.

Page and Brin developed the link-based search further by comparing links of web pages to references in academic work (Vise \& Malseed, 2006). The more references lead to one particular academic work, the greater the importance of the work. The same should be true for websites. Quickly, both inventor entrepreneurs began to program a link rating system which gave more weight to web-pages that were highly mentioned by links. In other words, websites with high traffic appeared as top searches while lowtraffic websites appeared in a lower position. A radical new invention was born. Page and Brin embedded the algorithm into their first official search program "BackRub", an immediate success. In a couple of weeks, it was used by thousands of Stanford students on campus. Only a few months after their invention, Page and Brin decided to rename their invention in what would become a world known brand, Google.

Revisiting this radial new invention with the LTS approach, Page and Brin can be considered independent inventor entrepreneurs. Both did not work for a Large Technical Systems, in this case major internet platforms such as Yahoo or Microsoft. Furthermore, their radical idea was repeatedly rejected as unfitting. As an illustration, in 1997 Yahoo which was worth multi-million dollar at the time, was offered the Google license for $\$ 500.000$. Yahoo declined, arguing that this search engine was too efficient and would lead users away from the platform itself. Google would run counter to their business model which was, very similar to Microsoft, to create a platform that would keep the users onsite for as long as possible. However, labelling Brin and Page as pure independent inventor entrepreneurs according to Hughes' description would also not capture the complete picture. Stanford University provides inventor entrepreneurs with the freedom to research ideas and inventions unfitting for other Large Technical Systems. Nonetheless, Stanford University also provides basic resources by supporting start-ups like 
Google financially on the condition of them receiving stocks in the case of a successful launch later during development (Vise \& Malseed, 2006).

\subsection{Development Phase}

Once the planted seed of a radical new invention can flourish, it proceeds into its developmental phase. According to Hughes' LTS approach, within the developmental phase, the invention develops a system structure and its first related components (Bijker, Hughes, Pinch, 2012). The new system is used by or is available to a larger group of consumers. It adapts to social economic and political characteristics of its environment. The inventor entrepreneur is still in charge of the system, yet the managing power is often distributed. Hughes suggests the inventor entrepreneur can be replaced or substituted by the management entrepreneur who has a further guiding position. Furthermore, during this phase, the system must encounter its first reverse salients. In many new technical systems, the most prominent first reverse salient is financial support. Up to this point, the system grows metaphorically in the dark. It is recognized by few, but not perceived as the LTS it is going to be. Hughes suggests, if the new system is offered to already existing Large Technical System's it is, similar to a radical new invention, rejected as an unfitting component. Finding investors who support the early system is therefore vital and of upmost importance.

After the radical new invention Backrub/ Google was founded, it did however not jump directly into the development phase. At that time, it was not even clear if their radical new invention would develop a system structure, which would add new components. New physical artifacts in the form of self-build computer systems were added to fulfill the demands of their ever more resource and energy-hungry program (Vise \& Malseed, 2006). This extension happened to such an extent that in mid-1997 Pages' dorm room was rebuilt into a server room. Yet, the inventor entrepreneur kept Google below its potential. Available under its first domain "google.stanford.edu", the search engine soon enjoyed prominence far beyond campus borders. Search demands were too high to be handled by two PhD student with a few computers. However, Page and Brin were skeptic about providing their radical new invention the needed system structure to fulfill its demands. They hesitated to launch Google's development stage. Even though from today's perspective this hesitation seems to be incomprehensible, in the late 90's Pages's and Brin's concerns were very valid. New internet services emerged everywhere and generated much traffic in little time. Yet, successfully attracting a large popularity did not automatically imply financial success. Multiple new internet companies were able to acquire a large share of public attention but went out of business because they were not profitable. Investing in an internet company was therefore risky which Page and Brin, in close contact to the tech industry, were aware of. Internet companies seemed to allow revolutionary ideas no one paid for. Simultaneously, their unsuccessful effort to sell the Google license underpinned their skepticism.

Regardless of the uncertainty, Google arrived at the developmental phase when the system builder Page and Brin followed an advice from the Yahoo co-founder David Filo. With a credit of 100.000 \$US from a private investor, Brin and Page founded the company Google Inc. and moved into their first office in Palo Alto, California in 1999 (Auletta, 2010). Immediately, the search engine flourished and continuously added new technical and non-technical components to its system structure. Page and Brin massively extended Google's server system. Even though their financial backup would allow to buy a professional large server system from IBM, Google continued to purchase and rebuild many weaker, yet cheaper, computers into a single powerful unit run by the open source system Linux. Besides avoiding higher costs, this strategy had 
the further advantage of not having to rely on external components. The servers fueling Google's searchengine were in complete control and ensured the autonomy of Google. Non-technical components were added in the form of an exclusive team of engineers selectively chosen by Page and Brin to improve the search engine and to keep the server system running. Everything indicated further growth.

In mid-1999, Google was able to generate 500.000 search requests on an average day and started to compete with other search engines such as AltaVista and even Yahoo, regarding the size of their searchindex (Vise \& Malseed, 2006). The former project of two PhD students grew into a company with 40 employees. But Google did not stop there. Though web-search was the priority for Brin and Page, the two inventor entrepreneurs intended to extend their now system structure beyond initial invention. For example, in 2000 the Google image search was integrated into the regular Google search. This was the first time a search engine incorporated this.

However, guided by two inventor entrepreneurs with a background in engineering, the system arguably grew without a precise plan or business strategy. The system builders Page and Brin followed the first and foremost goal which was to optimize the system. This soon resulted in the company's encounter with its first reverse salient - finance. To recapitulate, the early success of Google can be boiled down to two aspects. Firstly, and unmistakably, Google delivered a better search service that its competitors. Secondly, Page and Brin strongly opposed conventional advertising. Unlike other websites, Google did not propagate flashy imagery and advertisement. On the one hand, this made the website incredibly fast, but on the other hand, undermined one of the most important financial pillars of web market at the time. The alternative, to charge people money for using their service, would not have worked either as users, even more than today, were extremely skeptical about paying for digital services. In other words, Google had no idea in which direction the system was supposed to go or how to make money in the first place. Google was stuck in the developmental phase.

The reverse salient was declared as critical problem when Google slowly ran out of its financial backup of 20 million dollar provided by early investors. As Hughes' LTS approach suggests in this phase, the system needed to adapt to the market. (Bijker, Hughes, Pinch, 2012) Hence the reverse salient was approached in a twofold way.

Firstly, the system guidance was extended by a third leading member, Eric Schmidt, whom can be considered as manager entrepreneur. Unlike Page and Brin, Schmidt had a background in management and was the former CEO of the software and service company "Novell, Inc.". Even though the main guiding force was in the hands of the two initial System Builders, Schmidt was supposed to introduce the first business plan to make the company sustainable. (Vise \& Malseed, 2006)

Secondly, to overcome the reverse salient of finance, Google introduced in 2001 a conservative invention called "AdWords", an advertising program associated with search. (Wiesend, 2015) The idea was simple. By associating key words with public sales, Google managed to link advertising to the user directly. In doing so, Google held online auctions where advertisers could bet on specific words such as names of car brands or public products in general. Such words were sold in a price range from a few cents up to more than 15 US\$ per click. If a user searched for a sold word, the advertising of a company which owned the word appeared in a small box next to the search results. Additionally, "AdWords" was subsidized by the so call "Cost-per-Click" concept. Advertisers were only charged if the user clicked on an advertiser's link. The more an advertisement was followed, the higher it also appeared in the search results. This way, Google revolutionized and democratized the advertising market at the same time. Companies worldwide were able to display their products for a relatively small amount of money. Massive PR budgets which 
could only be provided by the most successful companies were no longer needed. The system builders had found a technical component to overcome its most critical problem. For the first time in 2001, Google could make a profit of $\$ 7$ million on revenues of $\$ 86$ million which in the next year even jumped to $\$ 100$ on revenues of $\$ 439$ (Auletta, 2010).

This invention came just in time. Already one year before, the so-called "Dot-com bubble", i.e. speculative investments due to a tremendous overvaluation of internet companies due to, started to bust. Many internet companies which did not find a financial pillar crashed. Yahoo, which was one of Google's largest competitors up to this point, lost not only much of its stock value but also many of its users, especially to Google. The technical system by Page, Brin and Schmidt, however, was barely affected by the financial disaster and even came out of the crisis better informed. Finally, Google was given the unique opportunity to establish market dominance, best depicted when Yahoo gave in completely and embedded Google's search engine into their own web-portal in 2001. According to Hughes, the system had passed the developmental phase (Vise \& Malseed, 2006).

\subsection{Innovation Phase}

Hughes argues, if the system overcomes its reverse salient, it approaches the innovation phase (Bijker, Hughes, Pinch, 2012). Thus, the system is ready to enter the market and to prevail in competition against other LTSs. By entering the market, the complexity established up to this point is revealed. Both the competition, as well as the public, become aware of the massive network of components which developed within the two previous phases. The process of revelation takes mostly place when a technical system goes public. Thus, not only its complexity is revealed, but also its financial worth and turnover. At this point, the system builder strives to increase the size of the system and decrease the influence of factors which are not in control of the system. Further components are added relating manufacturing, sales and service facilities. Such new components can be part of the initial company, but also be entire new companies which are related to the system. Overall, the system attempts to create a dominating market position. The radical new invention from the beginning slowly acquires the shape of a Large Technical System. Hughes suggests that, in this phase the influence of the inventor-entrepreneur slowly decreases, while the influence of the manager-entrepreneur increases.

Google entered the stage of innovation on the 19th of August 2004, when the system builder affirmed the companies worth and turnover and thus revealed its complexity. The public became aware that Google not only created a completely new market in advertising but also that the company was already deeply rooted in it.

The step of going public was mainly initiated by Schmidt and against the will of Brin and Page (Vise \& Malseed, 2006). According to Schmidt, Google needed more investment capital, and going public would be the only possible solution. The two inventor entrepreneurs, however argued against Schmidt by claiming that going public would reveal the true trajectory of Google. Google's potential competition would find out how much money is behind combining search with advertising and would join the new market. Furthermore, going public would imply that the company was in public hands. Hence, Page and Brin feared that short time growth- the immediate satisfaction of Google stock buyers - would become most essential, restricting the inventor-entrepreneurs from risky investments.

After a long debate, Google again took a rather unconventional turn. Again, not relying on external environments, in this case the Wall Street stock market, Google created something unique at the time: 
its own stock selling component, Google stocks. Shareholders could buy shares as usual, but Google reserved the rights of deciding which investments it was going to make. In other words, Google was in public hands, yet the system builders still had full control over the system. Page, Brin and Schmidt were able to invest in components which might not be of interest to the investors in the short run, but which eventually benefited the system in the long run. Thus, Google introduced and strongly invested in additional components such as "Google Books" (2004), "Google Scholar" (2004) and "Google News" (2006). Even though the company's unusual behavior rose a lot of suspicion and skepticism in the American business world, the plan turned out as planned. During the first day on the stock market, the Google stock reached $\$ 108,31$, on the next day jumped even to $\$ 200$ and finally rested at approximately $\$ 300$. Within a few days, hundreds of Google employees became millionaires and the initial system builders, billionaires. The day Google went public, Google's public offering - an official document which declares a company's offering of stock - wrote: "We began as a technology company and have evolved into a software, technology, advertising and media company, all rolled into one." (Auletta, 2010, p. 186). Hughes could not have given a better description of a Large Technical System.

However, one aspect of Hughes innovation phase is unfitting in describing the development of Google. For Google, it is not the case that the inventor-entrepreneur is substituted by the managerentrepreneur. Though Schmidt kept a major advisory role, the system remained in the hands of Page and Brin. The innovative and inventory characteristics of the inventor-entrepreneur were supposed to pilot the technical system further on.

\subsection{Google - a Large Technical System?}

Yet, can Google be characterized as a Large Technical System already in the year of 2005? To provide an answer to this question, I considered the fundamental structure of an LTS described in the beginning of this paper. Clearly, Google established a system structure of a variety of components. It consists of physical artifacts in the form of server systems which power the engine and web products, organizational structures such as previously named web products (Google Search, Google News, Gmail and Google maps), human components in the form of employed engineers, as well as institutional elements such as a regulatory framework opposed by the system builders Brin, Page and Schmidt and a system culture which will be evaluated in the "momentum" section. All components aim for the system goal of improving the World Wide Web, attracting user attention and expansion. Page, Brin and Schmidt are in close relation to the system. They steer its direction but also depend on its performance. The system consciously attempts to incorporate environmental factors, which is seen as an ongoing attempt to achieve and defend market domination in web search. With this in mind, I conclude that labelling Google as a LTS is justified.

\section{Competition and Consultation}

Nevertheless, having described the emergence of an LTS, Hughes approach does not reach its resting point here. Instead, his research methodology changes from a historically descriptive approach in which identifiable actors are in charge of the system towards an analytical frame in which structural features and tensions of the system are analyzed. Too many internal and external factors influence the system at this stage of development which is why an explicate analysis only in relation to system builders in no longer possible. According to Hughes, the new LTS enters competition and consultation, a vaguely defined phase that aims to describe the systems dynamics. As the phase's name suggests, mature LTSs have the 
tendency to grow though the means of competing and consolidating. Within my analysis, I have particularly regarded the competition and consultation's sub-concepts of momentum, transfer and technical style which are most characteristic for this phase. In my analysis, momentum describes the force an LTS acquired during its later evolution while transfer, largely determined by the system's technical style, limits the demonstrated force, pointing towards factors which have further influencing power onto the system. (Bijker, Hughes, Pinch, 2012)

\subsection{Momentum}

Momentum suggests that the LTS becomes less dependent on its environment. (Mayntz \& Hughes, 2005) The interconnection of components created the appearance of autonomy. In other words, the Large Technical System acquired such a mass and velocity that inner dynamics seem to drive the course of development. The more components are involved into the system structure, the more momentum increases. To an outsider, it may feel as it the LTS is not affected by external environmental factors. It appears as if the system can no longer be controlled by external forces. A relevant sub-concept of momentum is what Hughes (1993) calls system culture. A system culture is the mindset which the system is guided by. It is imposed by the system builders to elucidate what is rational and desirable for development. To put it another way, the system culture can be compared to a company's ethical code.

Within the frame of the LTS approach, Google eventually entered the competition and consultation phase between 2002 - 2006. After a successful deal with AOL in 2002 in which Google became the web platform's official search engine, and with the additional defeat of Yahoo, Google seemed to gain momentum by having obtained a dominant market position in web search (Vise \& Malseed, 2006). No other Large Technical System, neither web platform, hard- or software provider, nor search engine seemed able to compete with Google. The LTS's sheer market dominance is best illustrated by showing its competition with the multinational technology company Microsoft. Exactly as Page had foreseen, going public came at the cost of drawing attention to the business's success formula. Everyone knew that the system builders managed to combine web search and advertising to create a completely new market. Microsoft, famous for bringing the computer to nearly every household, planned to join the race of searchengines. Just as it did with the previous web search engine "Netscape", Microsoft tried to surpass Google by introducing a similar search engine, intending to outplay its competition with a larger market share. In a press conference in the fall of 2004, Microsoft released a statement that their own search engine had surpassed Google's search index by one billion websites, now enabling users to search the web within a search index of five billion websites. This step was extremely cost intensive for Microsoft. Web search was a business branch which previously did not gain massive attention and was not even financially beneficial without Google's "AdWords". Microsoft's idea however turned out to be more difficult than expected. A few days after Microsoft's official announcement, Google also disclosed a press release, stating their former search index doubled and encompassed now almost the entirety of the internet. Eight billion websites were now available to the user. Google's momentum which it established through its network of technical and no-technical components, programs, algorithms and server structures shattered its competition.

But momentum can also be perceived without focusing on Google's most profitable share in the internet market. According to Hughes, momentum is obtained when an LTS becomes less dependent on its environment by incorporating it into the system (Bijker, Hughes, Pinch, 2012). Zooming out, Google did not only stick to its initial environment of web search but has grown in multiple directions which are 
barely related to its core idea. In 2001/2002. Google introduced Google News, an attempt to centralize news stories from all over the world on one page. In 2001 Google set off Google Earth, a global attempt to photograph the entire world, followed by Google Maps in 2004. Yet, most illustrating for Google's expansion to different environments is "desktop search". "Desktop search" was a program which could be downloaded to a Microsoft computer and search the computer better than its initial operating system (Vise \& Malseed, 2006). A further embarrassment for Microsoft. Clearly, these are just three examples of web search unrelated components which would need further investigation by themselves, yet they demonstrate the enormous velocity Google gained during its evolution.

I argue, however, that especially the appearance of momentum has a different source despite the system's evolution and growth. Media has a major share in creating Google's image of being unstoppable. Magazines and newspapers like the "The Wall Street Journal" or "Fortune", must reads in the American business world, published headlines such as "Why Google Scares Bill Gates" or "Living in the Google; Welcome to Microsoft Nightmare" (Vise, Malseed, 2006, p.253). Doubtless, Google became an LTS which was almost impossible to compete with, but such headlines stressed this notion even further.

Yet, it is important to stress, Google is aware of its momentum and even further, which impression momentum can have on the external environment. If much power is concentrated in one place, people tent to become suspicious about how such power is used. As I will demonstrate later this was not different for Google. Google attempted to oppose this notion by developing a highly valued system culture. The motto "Don't be evil", introduced in 2000/2001 was supposed to influence every inventor's ideas and to be of guidance to Google's business strategy (Schmidt, 2014). Surely, this motto can be critiqued as Google's naïve and idealistic attempt of "making the world a better place", especially as it was still up to Page and Brin to decide what is "evil". Nonetheless, no other company did make their motto to such an important part of their business culture.

To recapitulate, within the phase of competition and consultation Google acquired momentum. From an outsider's perspective, it seems as if Google's success was unstoppable. The system continuously grew without the restriction of any actor. Yet, is that true? In the following I suggest, even though Google gained the notion of momentum, it did not become technological deterministic. To understand that Google's momentum is influenced by further factors, I rely on the concept of transfer.

\subsection{Transfer}

The second concept to which Hughes pays a lot of attention within the phase of competition and consultation is transfer (Bijker, Hughes, Pinch, 2012). The concept of transfer describes the system's adaption to new geographical, social or political factors. While the system is growing and expanding into new environments, e.g. new countries, new markets, new legislations or social norms it must also deal with such influencing factors. The function of components in a different environment might be influenced. Components might not be able to operate in the same way. Transfer into a new environment can either be successful or unsuccessful. The LTS either adopts to the new environment or the system fails and cannot sustain in the environment. The success of an LTS's transfer is determined by its technical style. The concept of technical style describes the unique characteristic of a Large Technical System and its components e.g. its legal ideas, organizational models and artifacts. During transfer, the technical style of a system might change but also other environmental factors such as participants of the market can adapt to an LTS technical style. Hughes included the framework of transfer to oppose the notion of 
technological determination due to momentum by suggesting that also an LTS is open to the social shaping of technology. (Mayntz \& Hughes, 2005)

Google's notion of transfer can be analyzed in two ways. Firstly, as Hughes intended in his original approach, when the company moves to a different environment in a geographical sense, it needs to adapt its system style. Secondly, if the company adds new components to the system, the system's technical style needs to be transferred onto such components as well. Yet, before evaluation how the concept of transfer can be assigned to Google, I need to clarify how I understand Google's technical style. In alignment with the LTS approach, I consider Google's second revolutionary invention "AdWords" as its manifestation of technical style. Since then, Google has been collecting data to match it with user related advertising.

During Google's competition and consultation phase, transfer in a geographical sense was a relatively minor problem. As is natural for an online platform, Google was able to operate on a global scale from the very beginning. The system did not have to alter its style to appeal to users from different cultural and geographical environments. The only minor system change was its translation to the respective countries' national languages (Vise \& Malseed, 2006). However, I do not consider that as an alternation of the system's style because the system was still able to run with and on its traditional components. Furthermore, as the internet was unregulated by political actors to a wide extent, Google as large technical system did not have to fear any law violations. Some publisher's campaign to improve the copyright, for instance, was largely ignored and only gained little public and institutional attention. Everything within the reach of the possible was permitted.

However, focusing my analysis on the transfer of Google's technical style onto its components, Google recognized quickly that this form of transfer was unexpectedly problematic. In the spring of 2004, Google introduced "Gmail" a free e-mail service which was supposed to be the counter draft to the marketdominating e-mail services by Microsoft and Yahoo. With a data storage of one gigabyte - at the time a massive amount - Google wanted to provide its customers with the comfort of not having to delete any mail anymore. To make the component profitable, Google transferred the same technical style from its search engine to its new e-mail service. Thus, Google analyzed what their users were typing and in accordance provided relevant advertising, arguably all for the benefit of the user. However, this transfer did not only backfire. It immediately became a reverse salient, putting the entire component "Gmail" into question. Knowing that Google Was scanning, categorizing and collecting data for further analysis generated a public outcry. Suddenly, privacy issues became an important point of discussion. People began to ask how Google search and "Gmail" were connected and what data would be stored by it. Google generated sensible data for commercial purposes. Already that was seen controversial, but activists also began to worry, what could happen, if such data fell into the hands of third parties. (Auletta, 2010)

The public discourse which was initiated due to this unsuccessful transfer went completely against their established system style. If Google wanted to maintain its momentum and scale of growth it needed to maintain its system style and hence rely on user data without any restrictions. Yet, if the users became unwilling to share their data, Google's system style could not be maintained, and if it did not adapt in a certain way, it would eventually cause Google to lose its momentum.

Noticeably, this interpretation of transfer and momentum guides the intention to a factor which massively influences the LTS yet finds not much attention in Hughes initial Large Technical System approach. Refocusing my research on this factor will bring further understanding about how momentum might be overcome - how the expansion of an LTS might be limited. 
I will again turn to Google and its behavior as an LTS. Furthermore, I need to complete Hughes's initial Large Technical System approach by adding one guiding component besides the system builder. Especially by analyzing contemporary internet companies, the user as a social actor is particularly important. Taking a social constructivist stance, I argue that the user and more specifically the user's favor decide if a system can pass Hughes's described stages of development. The user, even more than the system builder, decides about the fate and future direction of the LTS. The user is not necessarily bound to an online service, especially if the service is free of cost in the first place. Thus, if the systems product or service does not appeal to the user, he will no longer support it. If the company does not provide the right service, users will immediately switch to a different one, reducing the company's income (Schulz, 2017).

The additional concept of the user will finally help to understand why Google emerged as Hughes predicted in his Large Technical System approach. It also explains why Google as Large Technical System does not behave in the way as predicted by Hughes's last phases by not opposing radical new inventions but embracing them.

During Google's invention phase and throughout its development and innovation, Google's future momentum and power was not obvious to the user. Google search was revolutionary and provided a handy tool for internet activity. It was free and provided a better product than its competitors. No user had concerns about the little, helpful search tool. How could it do any harm? With the users' blind support, Google was able to pass the described phases of invention and development.

Nonetheless, this does not mean that Google was uninformed of its users and their power. Since the very beginning, Google Inc. and especially its system builders, put much work in the interaction with its users. As an illustration, from 1998 to 2011 Page and Brin wrote a monthly newsletter named "Google Friends" in which they informed users about their latest updates, future goals and had a section for user feedback. Google never developed completely in the dark and strongly engaged in user discourse. Inspired by the academic tradition of Stanford University, Page and Brin regularly conducted experiments and tests to find out how their users behaved and how the search engine could be improvised accordingly (Vise \& Malseed, 2006). This way, for instance, in 1999 the System Builders discovered that their almost blank web page did not simplify its usage, but rather had a confusing effected. Some users starred at the page for more than five minutes. When asked later, they replied they were waiting for the website to fully load. This is the only reason why Google included a footer with contact details. Furthermore, it demonstrates perfectly the users' influence on the system.

The close user contact helped Google to imply a sense of transparency. In the eyes of the users, Google delivered a service which can be consumed, but nothing more. Combined with the strongly propagated system culture "Don't be evil", Google established an image of a user-friendly company whose primary intention was to improve the internet and later the world as such. This user image was of high importance when Page, Brin and Schmidt overcame their reverse salient finance, added the component "AdWords" in 2004 and pushed their company into the innovation phase. The user's carefree deployment of data was Google's financial fundament (Auletta, 2010).

However, this user image was firstly tested with Google's failed transfer of Gmail and its successful stock market launch. Eventually, the user became aware of the system's influencing power and complexity considered as momentum. People became suspicious of Google's monopoly position. Even though Page and Brin repeatedly assured their good intentions, no one could guarantee that their promises were 
fulfilled. Slowly, concerns about data protection and privacy issues entered the public's consciousness. The reverse salient which first manifested due to Gmail remains a critical problem until today. Providing a free and competitive service keeps Google in its current dominant position. However, the louder the critical voices become, the harder it will become for Google to validate its technical style.

So far, it remains unclear how or if Google will overcome this reverse salient.

Google was aware of its dominant but instable position. For years, the company has tried to establish a new financial basis. In doing so, Google opposed the path suggested by Hughes. Instead of relying heavily on its only financial pillar and rejecting every unfitting component, Google sought innovation. Radical new inventions were not rejected, but highly supported. Google's unique stock allowed high risk inventions and even Google's work plan suggests the embracement of the radically new. Twenty percent of a Google-employee's work time, or one day each week, is supposed to be devoted to a project of the employee's choice (Schmidt, 2014). It remains to be seen if the strategy of innovation will provide a solution for Google's critical problem and the user can be continuously be convinced.

\section{Conclusion}

I have demonstrated that Google can successfully be labeled a Large Technical System including the concepts of momentum and transfer. The Large Technical System approach can be helpful to understand the origin and growth of complex networks and systems. The general framework provides a useful tool to conceptualize the company's development and power even though not all elements of the Large Technical System approach are applicable to Google. The findings reveal that, the domination of power of the manager-entrepreneur in innovation phase or behavior of the mature LTS in the competition and consultation phase did not take place as suggested by Hughes.

The concepts of momentum and transfer can furthermore be applied to comprehend the system's expansion after attaining a large technical structure. Hughes' concepts vividly illustrate the dynamics of an LTS, but only the consideration of the user suggests that momentum is just a notion and that a system's decline can arise faster than expected. In essence, it is true that Google started out with a small revolutionary idea and became one of the most powerful companies in the world. Yet, it is not excluded from external influences and its momentum might not be eternal. The user, more than any other actor, decides the company's future.

Although according to the LTS criteria I established previously in my paper, the analysis was restricted by several factors. I had to restrict my analysis by not involving the concept of load factor (Joerges, 1996). Although, this concept appears repeatedly throughout the competition and consultation period and is described as "the ratio of average system output to maximum output over a given period" (Joerges, 1996, p. 14), there was no valid opportunity to involve the concept in the analysis of Google.

Even though my initial research question, namely what extent the theoretical concept of Large Technical Systems (LTS) can provide fruitful insights into the complexity and success of this internet platform, is answered positively and validates the LTS approach, it paves the way for a variety of subsequent questions. After the Large Technical System arrives at a dominant, mostly global position, Hughes' analysis stops. However, he states that LTSs are in constant change, so how do they maintain their dominant position? Furthermore, Hughes' describes a system evolution only within the frame of system growth. Components are added to a system and are later attached to by other components - the network structure. Yet, his approach does not explain what it means if one component is omitted. Moreover, it is not 
elaborated if some components are less essential to the system than others and who determines the value. Has Google search the equal value such as newer branches of the company?

Following research into Google's development within the frame of LTS might be complemented by the research of Arne Kaijser (1995). Noticeably, Kaijser turned away from a heavy focus on individual guiding actors and instead payed further attention to the links of an LTS's components. Within his theory of system coupling, his main interest is in linking LTS with further institutional actors. Kaijser seeks to find answers on what institutional and governing frameworks steer and how they influence an LTS, especially if the mature LTS spreads across country borders. In the case of Google, his approach is of special interest. Within recent years, Google as a company gained an increasingly ambivalent name across different nations. Though Google's services are massively used on a global scale, governments start to restrict Google's power. To illustrate, in May 2014, the Court of Justice of the European Union asserted the "right to be forgotten" as fundamental principal into EU law (Zuboff, 2019). Enforceable since May 2018, the General Data Protection Regulation enables EU citizens to request service providers to erase personal data concerning them under certain conditions (EU 2016/679, Art. 17). This implies the right not to be shown among Google search results any longer. This example shows that institutions and governments begin to adapt laws to the previously unregulated realm of the internet, but also that companies such as Google are no longer independent of any regulatory constraints. Further research in relation to Kaijser's work could, for example, investigate how these altered boundary conditions might influence an LTS's development. The thus obtained findings could hence be used to extend the here established application of the LTS framework on Google by addressing the question of how the future evolution of Google might look like.

\footnotetext{
16 Research Papers
} 
Auletta, K. (2010). Googled: The end of the world as we know it. London: Virgin Books.

Baskin, B., Brashars, J., \& Long, J. (2007). Google talking. Rockland, MA: Syngress.

Bijker, W., Hughes, T., \& Pinch, T. (2012). The social construction of technological systems: New directions in the sociology and history of technology (Anniversary ed.) [Anniversary ed.]. Cambridge, Mass.: MIT Press.

Burns, C., \& Sauers, M. (2014). Google search secrets. Chicago: Neal-Schuman/American Library Association.

De Vynck, G. (2019). Alphabet Profit Margins Slide as Google Costs March Upward. Retrieved June 11, 2019 from https://www.bloomberg.com/news/articles/2019- 02-04/alphabet-profit-marginsslide-as-google-costs-march-upwards

Gökalp, I. (1992). On the Analysis of Large Technical Systems. Science, Technology, \& Human Values, 17(1), 57-78. Retrieved June 11, 2019 from http://www.jstor.org/stable/689850

Guermeur, D., \& Unruh, A. (2010). Google app engine java and gwt application development. Birmingham: Packt Pub.

Holden, G. (2008). Go google: 20 ways to reach more customers and build revenue with google business tools. New York: AMACOM.

Hughes, T. P. (1993). Networks of Power: Electrification in Western Society. Baltimore, MD: John Hopkins University Press.

Hughes, T. P. (2000). Rescuing Prometheus: Four Monumental Projects That Changed the Modern World. New York: Vintage Books.

Joerges, B. (1996). Large Technical Systems and the Discourse of Complexity. In Complex Technical Systems, Swedish Council for Planning and Coordination of Research (pp. Stockholm: Affärs Litteratur 1996.

Kaijser, A. (2005). How to Describe Large Technical Systems and Their Changes over Time? Urban Transport Development, 12-19. doi:10.1007/3-540-27761-7_3

Kaijser, A., \& Vleuten, E. V. (2006). Networking Europe: Transnational infrastructures and the shaping of Europe, 1850-2000. Sagamore Beach: MA: Science History Publications.

Kaijser, A. (1995). Nordic energy systems: Historical perspectives and current issues. Canton Mass.: Science History Publications/USA.

Lowe, J. (2009). Google speaks : Secrets of the world's greatest billionaire entrepreneurs, sergey brin and larry page. Hoboken, N.J.: John Wiley \& Sons.

Mayntz, R., \& Hughes, T. P. (2005). The Development of large technical systems. New York: ACLS History E-Book Project.

Muniesa, F. (2015). Actor-Network Theory. International Encyclopedia of the Social \& Behavioral Sciences (Second Edition), 80-84. doi:10.1016/b0-08-043076-7/04743-4

Regulation (EU) 2016/679 of the European Parliament and of the Council of 27 April 2016 on the protection of natural persons with regard to the processing of personal data and on the free movement of such data, and repealing Directive 95/46/EC (General Data Protection Regulation), Official Journal of the European Union L119, 4.5.2016. 
Ryan, J. (2013). A history of the Internet and the digital future. London: Reaktion Books.

Schmidt, E. (2014). Google: How google works (First ed.). New York: Grand Central Publishing.

Schulz, T. (2017). Was Google wirklich will. Wie der einflussreichste Konzern der Welt unsere Zukunft verändert - Ein SPIEGEL-Buch. München: Penguin.

Vise, D. A., \& Malseed, M. (2006). Google story. Paris: Dunod.

Wiesend, S. (2015). Page Rank, Adwords und Milliardengewinne: Die Geschichte von Google. Retrieved February 03, 2015 from https://www.computerwoche.de/a/die-geschichte-von-google,3064145

Zuboff, S. (2019). The age of surveillance capitalism: The fight for a human future at the new frontier of power. London: Profile Books. 